\title{
POTENTIALS OF A MARKOV PROCESS ARE EXPECTED SUPREMA*
}

\author{
HANS Föllmer ${ }^{1}$ And Thomas KNisPel ${ }^{1}$
}

\begin{abstract}
Expected suprema of a function $f$ observed along the paths of a nice Markov process define an excessive function, and in fact a potential if $f$ vanishes at the boundary. Conversely, we show under mild regularity conditions that any potential admits a representation in terms of expected suprema. Moreover, we identify the maximal and the minimal representing function in terms of probabilistic potential theory. Our results are motivated by the work of El Karoui and Meziou (2006) on the maxplus decomposition of supermartingales, and they provide a singular analogue to the non-linear Riesz representation in El Karoui and Föllmer (2005).
\end{abstract}

Mathematics Subject Classification. 31C05, 60J25, 60J45.

Invited paper accepted September 2005.

\section{INTRODUCTION}

For a nice Markov process such as Brownian motion on a bounded domain of $\mathbb{R}^{d}$, we consider the excessive function $u$ defined by the expected suprema

$$
u(x):=E_{x}\left[\sup _{0<t<\zeta} f\left(X_{t}\right)\right]
$$

of some function $f \geq 0$ observed along the paths of the process up to its life time $\zeta$. The function $u$ is excessive, and it is in fact a potential if $f\left(X_{t}\right)$ converges to zero as $t \uparrow \zeta$. Conversely, we show under mild regularity conditions that any potential $u$ admits a representation of the form (1) in terms of expected suprema.

In general, the representing function $f$ is not uniquely determined by $u$. We show that the maximal representing function is given by

$$
\underline{D} u(x):=\inf \frac{u(x)-P_{T} u(x)}{P_{x}[T=\zeta]},
$$

where the infimum is taken over all exit times $T$ from open neighborhoods of $x$ such that $P_{x}[T=\zeta]>0$. On the other hand, the minimal representing function is identified as

$$
\underline{D} u 1_{H^{c}},
$$

Keywords and phrases. Markov processes, potentials, optimal stopping, max-plus decomposition.

* Supported by the DFG Research Center MATHEON "Mathematics for Key Technologies" in Berlin.

${ }^{1}$ Humboldt-Universität zu Berlin, Institut für Mathematik, Unter den Linden 6, 10099 Berlin, Germany;

foellmer@math.hu-berlin.de; knispel@math.hu-berlin.de

(c) EDP Sciences, SMAI 2007 
where $H$ is the set of points $x$ such that $u$ is harmonic in some neighborhood of $x$.

Our discussion of the existence problem is motivated by the work of El Karoui and Meziou [7] and El Karoui [5] which involves a representation of a given supermartingale as a process of conditional expected suprema of some other process. Such a representation is of considerable interest, as illustrated by the financial applications discussed in [7]. Here we translate some of the key arguments in [5] into the setting of probabilistic potential theory. This is analogous to the non-linear Riesz representation

$$
u(x)=E_{x}\left[\int_{0}^{\zeta} \sup _{0 \leq s \leq t} f\left(X_{s}\right) \mathrm{d} t\right]
$$

in El Karoui and Föllmer [6] which can be seen as a special case of a general representation theorem due to Bank and El Karoui [1]; see also Bank and Föllmer [2] for a survey. In the Markovian setting, both (1) and (2) may be viewed as special cases of a representation

$$
u(x)=E_{x}\left[\int_{0}^{\zeta} \sup _{0 \leq s \leq t} f\left(X_{s}\right) \mathrm{d} A_{t}\right]
$$

with respect to a given additive functional $\left(A_{t}\right)_{t \geq 0}$ of the underlying Markov process. Indeed, in (2) the additive functional is given by $A_{t}=t \wedge \zeta$, and in (1) it corresponds to the random measure $\delta_{\zeta}$. So far, representation results with respect to additive functionals are only available under strong regularity assumptions which exclude the singular random measure $\delta_{\zeta} ; c f$. Knispel [8] for a discussion in the Markovian setting, where the random measure $\mathrm{d} A_{t}$ satisfies the conditions described in Remark 1.1 of Bank and El Karoui [1]. For this reason it seems useful to prove the existence of a representation for the case of the random measure $\delta_{\zeta}$ in the context of probabilistic potential theory. Moreover, the present paper contains new results related to the uniqueness problem which involve the harmonic points of the function $u$.

\section{Preliminaries}

Let $\left(X_{t}\right)_{t \geq 0}$ be a strong Markov process with locally compact metric state space $(S, d)$, shift operators $\left(\theta_{t}\right)_{t \geq 0}$, and life time $\zeta$, defined on a stochastic base $\left(\Omega, \mathcal{F},\left(\mathcal{F}_{t}\right)_{t \geq 0},\left(P_{x}\right)_{x \in S}\right)$. As in El Karoui and Föllmer [6] we introduce an Alexandrov point $\Delta$ and use the following assumptions:

A1) The process $\left(X_{t}\right)_{t \geq 0}$ is a Hunt process in the sense of [3] XVI.11 such that $\lim _{t \uparrow \zeta} X_{t}=\Delta$.

A2) The excessive functions of the process are lower-semicontinuous.

As a typical example, we could consider a Brownian motion on a bounded domain $S \subset \mathbb{R}^{d}$.

Let us denote by $\mathcal{T}(x)$ the class of all exit times

$$
T_{U^{c}}:=\inf \left\{t \geq 0 \mid X_{t} \notin U\right\} \wedge \zeta
$$

from open neighborhoods $U$ of $x \in S$, and by $\mathcal{T}_{0}(x)$ the subclass of all exit times from open neighborhoods of $x$ which are relatively compact. Note that $\zeta=T_{S^{c}} \in \mathcal{T}(x)$.

For a measurable function $u \geq 0$ on $S$ and a stopping time $T$ we use the notation

$$
P_{T} u(x)=E_{x}\left[u\left(X_{T}\right) ; T<\zeta\right]
$$

Recall that $u$ is excessive if $P_{t} u \leq u$ for any $t>0$ and $\lim _{t \downarrow 0} P_{t} u(x)=u(x)$ for any $x \in S$. 
Definition 2.1. An excessive function $u \geq 0$ will be called a potential of class (D) if, for any $x \in S$,

$$
\lim _{t \uparrow \zeta} u\left(X_{t}\right)=0 \quad P_{x}-a . s .
$$

and the family

$$
\left\{u\left(X_{T}\right) \mid T \in \mathcal{T}_{0}(x)\right\} \text { is uniformly integrable with respect to } P_{x} .
$$

Proposition 2.1. Let $f \geq 0$ be an upper-semicontinuous function on $S$. Then the function $u$ on $S$ defined by the expected suprema

$$
u(x):=E_{x}\left[\sup _{0<t<\zeta} f\left(X_{t}\right)\right]
$$

is excessive, hence lower-semicontinuous. Moreover, $u$ is a potential of class $(D)$ if and only if $f$ satisfies the conditions

$$
\sup _{0<t<\zeta} f\left(X_{t}\right) \in \mathcal{L}^{1}\left(P_{x}\right)
$$

and

$$
\lim _{t \uparrow \zeta} f\left(X_{t}\right)=0 \quad P_{x}-a . s
$$

for any $x \in S$.

Proof. 1) Upper-semicontinuity of $f$ ensures that $\sup _{0<t<\zeta} f\left(X_{t}\right)$ is measurable, and so $u$ is well defined as a measurable function on $S$. Since

$$
P_{t} u(x)=E_{x}\left[E_{X_{t}}\left[\sup _{0<s<\zeta} f\left(X_{s}\right)\right] ; t<\zeta\right]=E_{x}\left[E_{x}\left[\sup _{t<s<\zeta} f\left(X_{s}\right) \mid \mathcal{F}_{t}\right] ; t<\zeta\right]=E_{x}\left[\sup _{t<s<\zeta} f\left(X_{s}\right) ; t<\zeta\right],
$$

we see that $P_{t} u(x) \leq u(x)$ and, by monotone convergence, $\lim _{t \downarrow 0} P_{t} u(x)=u(x)$ for any $x \in S$, i.e., $u$ is excessive.

2) Suppose that $f$ satisfies the conditions (5) and (6). Then $u$ is finite on $S$. Recall that $\lim _{t \uparrow \zeta} u\left(X_{t}\right) \operatorname{ex-}$ ists $P_{x}-$ a.s. for any excessive function $u$. Take $T_{n}$ as the exit time from $U_{n}$, where $\left(U_{n}\right)_{n \in \mathbb{N}}$ is a sequence of relatively compact open neighborhoods of $x$ increasing to $S$. Since $0 \leq \sup _{0<t<\zeta} f\left(X_{t}\right) \in \mathcal{L}^{1}\left(P_{x}\right)$,

$$
0 \leq \lim _{n \uparrow \infty} u\left(X_{T_{n}}\right)=\lim _{n \uparrow \infty} E_{x}\left[\sup _{T_{n}<s<\zeta} f\left(X_{s}\right) \mid \mathcal{F}_{T_{n}}\right] \leq \lim _{n \uparrow \infty} E_{x}\left[\sup _{T_{n_{0}}<s<\zeta} f\left(X_{s}\right) \mid \mathcal{F}_{T_{n}}\right]=\sup _{T_{n_{0}}<s<\zeta} f\left(X_{s}\right) \quad P_{x}-a . s .
$$

for any $n_{0}$ due to the martingale convergence theorem, hence $\lim _{t \uparrow \zeta} u\left(X_{t}\right)=0 P_{x}-$ a.s. in view of our assumption (6) on $f$. Moreover, $\left\{u\left(X_{T}\right) \mid T \in \mathcal{T}_{0}(x)\right\}$ is uniformly integrable with respect to $P_{x}$ since

$$
0 \leq u\left(X_{T}\right)=E_{x}\left[\sup _{T<t<\zeta} f\left(X_{t}\right) \mid \mathcal{F}_{T}\right] \leq E_{x}\left[\sup _{0<t<\zeta} f\left(X_{t}\right) \mid \mathcal{F}_{T}\right]
$$

Thus $u$ is a potential of class (D). Conversely, if $u$ is a potential of class (D) then $u(x)<\infty$ due to condition (4), since $u(x) \leq \underline{\lim }_{n \uparrow \infty} u\left(X_{T_{\epsilon_{n}}}\right)$ for the exit times $T_{\epsilon_{n}} \in \mathcal{T}_{0}(x)$ from the open balls $U_{\epsilon_{n}}(x)$, where $\epsilon_{n} \downarrow 0$. Thus $f$ satisfies condition (5). Moreover, (6) follows from

$$
\varlimsup_{t \uparrow \zeta} f\left(X_{t}\right)=\lim _{n \uparrow \infty} \sup _{T_{n}<s<\zeta} f\left(X_{s}\right)=\lim _{n \uparrow \infty} E_{x}\left[\sup _{T_{n}<s<\zeta} f\left(X_{s}\right) \mid \mathcal{F}_{T_{n}}\right]=\lim _{n \uparrow \infty} u\left(X_{T_{n}}\right)=0 \quad P_{x}-\text { a.s. }
$$

where the second identity is obtained by a martingale convergence argument.

Our purpose is to show that, conversely, any potential of class (D) admits a representation of the form (1) in terms of some upper-semicontinuous function $f$ satisfying the conditions (5) and (6). 


\section{Existence of A REPRESENTING FUnCTION}

Let $u$ be a potential of class (D). In order to avoid additional technical difficulties, we also assume that $u$ is continuous. For convenience we introduce the notation $u^{c}:=u \vee c$.

As a first step in our construction of a function $f$ such that $u$ can be represented in the form (1), we consider the family of optimal stopping problems

$$
R u^{c}(x):=\sup _{T \in \mathcal{T}_{0}(x)} E_{x}\left[u^{c}\left(X_{T}\right)\right]
$$

for $c \geq 0$ and $x \in S$. Note that

$$
u^{c}(x) \leq R u^{c}(x)=\sup _{T \in \mathcal{T}_{0}(x)}\left(E_{x}\left[u\left(X_{T}\right) ; u\left(X_{T}\right) \geq c\right]+c P_{x}\left[u\left(X_{T}\right)<c\right]\right) \leq u(x)+c<\infty
$$

for any $x \in S$.

It is well known that the value function $R u^{c}$ of the optimal stopping problem (7) can be characterized as the smallest excessive function dominating $u^{c}$; see, for example [9], Theorem III.1. In particular, $R u^{c}$ is lower-semicontinuous due to our assumption A2). Moreover,

$$
R u^{c}(x) \geq E_{x}\left[u^{c}\left(X_{T}\right) ; T<\zeta\right]+c P_{x}[T=\zeta]
$$

for any stopping time $T \leq \zeta$, and equality holds for the first entrance time $D_{0}^{c}$ into the set $\left\{R u^{c}=u^{c}\right\}$; cf. for example [4], Theorem 2.76, or the proof of Lemma 4.1 in [6]. Redefining $D_{0}^{c}$ as $\zeta$ on $\left\{D_{0}^{c}<\zeta, u\left(X_{D_{0}^{c}}\right)<c\right\}$, we can rewrite the equality as

$$
R u^{c}(x)=E_{x}\left[u^{c}\left(X_{D^{c}}\right) ; D^{c}<\zeta\right]+c P_{x}\left[D^{c}=\zeta\right]
$$

where

$$
D^{c}:=\inf \left\{t \geq 0 \mid X_{t} \in A(c)\right\} \wedge \zeta
$$

is the first entrance time into the set

$$
A(c):=\left\{R u^{c}=u\right\}
$$

Note that $A(c)$ is closed since $R u^{c}$ is lower-semicontinuous and $u$ is assumed to be continuous.

We are now going to study the dependence of $R u^{c}(x)$ and of $D^{c}$ on the parameter $c$, in analogy to the discussion in El Karoui and Föllmer [6].

Lemma 3.1. For any $x \in S, R u^{c}(x)$ is increasing, convex and Lipschitz-continuous in c, and

$$
\lim _{c \uparrow \infty}\left(R u^{c}(x)-c\right)=0 .
$$

Moreover, the map $c \mapsto D^{c}$ is increasing and $P_{x}-$ a.s. left-continuous.

Proof. 1) Since $c \mapsto u^{c}(x)=u(x) \vee c$ is an increasing and convex function which satisfies $u^{c}(x) \leq u^{a}(x)+|c-a|$ for $a, c \geq 0$, monotonicity, convexity and Lipschitz-continuity of $c \mapsto R u^{c}(x)$ follow immediately from definition (7). Moreover,

$$
0 \leq \lim _{c \uparrow \infty}\left(R u^{c}(x)-c\right) \leq \lim _{c \uparrow \infty} \sup _{T \in \mathcal{T}_{0}(x)} E_{x}\left[u\left(X_{T}\right) ; u\left(X_{T}\right)>c\right]=0
$$

due to our assumption (4) on $u$. 
2) By monotonicity of the mapping $c \mapsto R u^{c}$ the sets $A(c)$ decrease in $c$, and so the stopping times $D^{c}$ are increasing in $c$. In order to prove the left-continuity of $c \mapsto D^{c}$, we fix an arbitrary $c>0$ and a strictly increasing sequence $\left(c_{n}\right)_{n \in \mathbb{N}}$ converging to $c$. Clearly,

$$
D^{*}:=\lim _{n \uparrow \infty} D^{c_{n}} \leq D^{c} \leq \zeta
$$

Let us verify the converse inequality $D^{c} \leq D^{*} P_{x}-$ a.s. By monotonicity of $R u^{c}(x)$ in $c$ we obtain the estimate

$$
0 \leq\left(R u^{c_{n}}-u\right)(x) \leq\left(R u^{c_{n+m}}-u\right)(x)
$$

for any $x \in S$, hence

$$
0 \leq\left(R u^{c_{n}}-u\right)\left(X_{D^{c_{n+m}}}\right) \leq\left(R u^{c_{n+m}}-u\right)\left(X_{D^{c_{n}+m}}\right)=0 \quad \text { on }\left\{D^{*}<\zeta\right\}
$$

since $A\left(c_{n+m}\right)$ is closed. By quasi-left-continuity of our Hunt process $\left(X_{t}\right)_{t \geq 0}$ and by lower-semicontinuity of $R u^{c_{n}}$ we get

$$
0 \leq\left(R u^{c_{n}}-u\right)\left(X_{D^{*}}\right) \leq \varliminf_{m \uparrow \infty}\left(R u^{c_{n}}-u\right)\left(X_{D^{c_{n}+m}}\right)=0 \quad P_{x}-\text { a.s. on }\left\{D^{*}<\zeta\right\}
$$

hence

$$
\left(R u^{c}-u\right)\left(X_{D^{*}}\right)=\lim _{n \uparrow \infty}\left(R u^{c_{n}}-u\right)\left(X_{D^{*}}\right)=0 \quad P_{x}-a . s . \text { on }\left\{D^{*}<\zeta\right\}
$$

by continuity of $R u^{c}$ in $c$. This shows $D^{c} \leq D^{*} P_{x}-$ a.s. on $\left\{D^{*}<\zeta\right\}$, and clearly we have $D^{*}=D^{c}$ on $\left\{D^{*}=\zeta\right\}$.

The function $c \mapsto R u^{c}(x)$ is convex, hence almost everywhere differentiable. The properties of the optimal stopping times $D^{c}$ allow us to determine the derivatives explicitly.

Lemma 3.2. The derivative $\partial^{-} R u^{c}(x)$ from the left-hand side of $R u^{c}(x)$ with respect to $c>0$ is given by

$$
\partial^{-} R u^{c}(x)=P_{x}\left[D^{c}=\zeta\right]
$$

Proof. For any $0 \leq a<c$, the representation (9) for the parameter $c$ combined with the inequality (8) for the parameter $a$ and for the stopping time $T=D^{c}$ implies

$$
R u^{c}(x)-R u^{a}(x) \leq E_{x}\left[u^{c}\left(X_{D^{c}}\right)-u^{a}\left(X_{D^{c}}\right) ; D^{c}<\zeta\right]+(c-a) P_{x}\left[D^{c}=\zeta\right] .
$$

Since

the previous estimate simplifies to

$$
u\left(X_{D^{c}}\right)=R u^{c}\left(X_{D^{c}}\right) \geq c>a \text { on }\left\{D^{c}<\zeta\right\},
$$

$$
R u^{c}(x)-R u^{a}(x) \leq(c-a) P_{x}\left[D^{c}=\zeta\right] .
$$

This shows $\partial^{-} R u^{c}(x) \leq P_{x}\left[D^{c}=\zeta\right]$. In order to prove the converse inequality, we use the estimate

$$
R u^{c}(x)-R u^{a}(x) \geq(c-a) P_{x}\left[D^{a}=\zeta\right]
$$

obtained by reversing the role of $a$ and $c$ in the preceding argument. Moreover, Lipschitz-continuity of $c \rightarrow$ $R u^{c}(x)$ yields $\cup_{a<c}\left\{D^{a}=\zeta\right\}=\left\{D^{c}=\zeta\right\}$ and this implies

$$
\partial^{-} R u^{c}(x) \geq \lim _{a \uparrow c} P_{x}\left[D^{a}=\zeta\right]=P_{x}\left[D^{c}=\zeta\right]
$$


Let us now introduce the function $f^{*}$ defined by

$$
f^{*}(x):=\sup \{c \mid x \in A(c)\}
$$

for any $x \in S$. Note that $f^{*}(x) \geq c$ is equivalent to $R u^{c}(x)=u(x)$ due to the continuity of $R u^{c}(x)$ in $c$.

Lemma 3.3. The function $f^{*}$ is upper-semicontinuous and satisfies $0 \leq f^{*} \leq u$. Moreover, $\lim _{t \uparrow \zeta} f^{*}\left(X_{t}\right)=0$ $P_{x}-$ a.s. for any $x \in S$.

Proof. In order to show that $f^{*}$ is upper-semicontinuous, we consider a sequence $\left(x_{n}\right)_{n \in \mathbb{N}}$ converging to $x$ such that $\lim _{n \uparrow \infty} f^{*}\left(x_{n}\right)=c>0$. Then $x_{n} \in A\left(c_{n}\right)$ for some sequence $\left(c_{n}\right)_{n \in \mathbb{N}}$ such that $c_{n} \uparrow c$. Since the decreasing sets $A\left(c_{n}\right)$ are closed, we obtain $x \in A\left(c_{n}\right)$ for any $n$, hence $f^{*}(x) \geq c$. The estimate $0 \leq f^{*}(x) \leq u(x)$ follows from $R u^{0}=u$ and $R u^{c}(x) \geq u^{c}(x)>u(x)$ for any $c>u(x)$. Moreover, $f^{*}\left(X_{t}\right)$ converges to zero as $t \uparrow \zeta$ since $f^{*} \leq u$, due to our assumption (3) on $u$.

We are now ready to derive a representation of the value functions $R u^{c}$ in terms of the function $f^{*}$.

Theorem 3.1. For any $c \geq 0$ and any $x \in S$,

$$
R u^{c}(x)=E_{x}\left[\sup _{0 \leq t<\zeta} f^{*}\left(X_{t}\right) \vee c\right]=E_{x}\left[\sup _{0<t<\zeta} f^{*}\left(X_{t}\right) \vee c\right] .
$$

Proof. By Lemma 3.2 and (10) we get

$$
R u^{c}(x)-c=\int_{c}^{\infty}-\frac{\partial}{\partial \alpha}\left(R u^{\alpha}(x)-\alpha\right) \mathrm{d} \alpha=\int_{c}^{\infty} P_{x}\left[D^{\alpha}<\zeta\right] \mathrm{d} \alpha .
$$

Since

$$
\left\{D^{c+\epsilon}<\zeta\right\} \subseteq\left\{\sup _{0 \leq t<\zeta} f^{*}\left(X_{t}\right)>c\right\} \subseteq\left\{D^{c}<\zeta\right\}
$$

for any $c \geq 0$ and for any $\epsilon>0$,

$$
\begin{aligned}
R u^{c}(x)-c & =\int_{c}^{\infty} P_{x}\left[D^{\alpha}<\zeta\right] \mathrm{d} \alpha \geq \int_{c}^{\infty} P_{x}\left[\sup _{0 \leq t<\zeta} f^{*}\left(X_{t}\right)>\alpha\right] \mathrm{d} \alpha \\
& \geq \int_{c}^{\infty} P_{x}\left[D^{\alpha+\epsilon}<\zeta\right] \mathrm{d} \alpha=R u^{c+\epsilon}(x)-(c+\epsilon) .
\end{aligned}
$$

By continuity of $c \mapsto R u^{c}$ we obtain

$$
R u^{c}(x)-c \geq \int_{c}^{\infty} P_{x}\left[\sup _{0 \leq t<\zeta} f^{*}\left(X_{t}\right)>\alpha\right] \mathrm{d} \alpha \geq \lim _{\epsilon \downarrow 0}\left(R u^{c+\epsilon}(x)-(c+\epsilon)\right)=R u^{c}(x)-c,
$$

hence

$$
\begin{aligned}
R u^{c}(x) & =\int_{c}^{\infty} P_{x}\left[\sup _{0 \leq t<\zeta} f^{*}\left(X_{t}\right)>\alpha\right] \mathrm{d} \alpha+c=E_{x}\left[\sup _{0 \leq t<\zeta} f^{*}\left(X_{t}\right)-\sup _{0 \leq t<\zeta} f^{*}\left(X_{t}\right) \wedge c+c\right] \\
& =E_{x}\left[\sup _{0 \leq t<\zeta} f^{*}\left(X_{t}\right) \vee c\right] .
\end{aligned}
$$


Moreover, we can conclude that

$$
R u^{c}(x)=\lim _{t \downarrow 0} P_{t}\left(R u^{c}\right)(x)=\lim _{t \downarrow 0} E_{x}\left[\sup _{t \leq s<\zeta} f^{*}\left(X_{s}\right) \vee c ; t<\zeta\right]=E_{x}\left[\sup _{0<s<\zeta} f^{*}\left(X_{s}\right) \vee c\right]
$$

since $R u^{c}$ is excessive, i.e., $R u^{c}(x)$ also admits the second representation in equation (12).

As a corollary we see that $f^{*}$ is a representing function for $u$.

Corollary 3.1. The potential $u$ admits the representations

$$
u(x)=E_{x}\left[\sup _{0 \leq t<\zeta} f^{*}\left(X_{t}\right)\right]=E_{x}\left[\sup _{0<t<\zeta} f^{*}\left(X_{t}\right)\right]
$$

in terms of the upper-semicontinuous function $f^{*} \geq 0$ defined by (11). Moreover,

$$
f^{*}(x) \leq \sup _{0<t<\zeta} f^{*}\left(X_{t}\right) \quad P_{x}-\text { a.s. }
$$

for any $x \in S$.

Proof. Note that $u=R u^{0}$ since $u$ is excessive. Applying Theorem 3.1 with $c=0$ we obtain

$$
u(x)=R u^{0}(x)=E_{x}\left[\sup _{0 \leq t<\zeta} f^{*}\left(X_{t}\right)\right]=E_{x}\left[\sup _{0<t<\zeta} f^{*}\left(X_{t}\right)\right]
$$

In particular we get

$$
\sup _{0 \leq t<\zeta} f^{*}\left(X_{t}\right)=\sup _{0<t<\zeta} f^{*}\left(X_{t}\right) \quad P_{x}-\text { a.s. },
$$

and this implies $f^{*}(x) \leq \sup _{0<t<\zeta} f^{*}\left(X_{t}\right) P_{x}-$ a.s. for any $x \in S$.

We have thus shown that $u$ admits a representing function which is regular in the following sense:

Definition 3.1. Let us say that a nonnegative function $f$ on $S$ is regular if it is upper-semicontinuous and satisfies the conditions

and

$$
\lim _{t \uparrow \zeta} f\left(X_{t}\right)=0 \quad P_{x}-a . s
$$

$$
f(x) \leq \sup _{0<t<\zeta} f\left(X_{t}\right) \quad P_{x}-a . s .
$$

for any $x \in S$.

Note that a regular function $f$ also satisfies the inequality

$$
f\left(X_{T}\right) \leq \sup _{T<t<\zeta} f\left(X_{t}\right) \quad P_{x}-\text { a.s. on }\{T<\zeta\}
$$

for any stopping time $T$, due to the strong Markov property.

Let us now derive an alternative description of the representing function $f^{*}$ in terms of the given excessive function $u$. To this end, we introduce the superadditive operator

$$
\underline{D} u(x):=\inf \frac{u(x)-P_{T} u(x)}{P_{x}[T=\zeta]}
$$

where the infimum is taken over all exit times $T$ from open neighborhoods of $x$ such that $P_{x}[T=\zeta]>0$. 
Proposition 3.1. The functions $f^{*}$ and $\underline{D} u$ coincide. In particular $x \mapsto \underline{D} u(x)$ is regular on $S$.

Proof. If $f^{*}(x)>c$ then $R u^{c}(x)=u(x)$, and in view of (8) this implies

$$
\begin{aligned}
u(x)-P_{T} u(x) & =R u^{c}(x)-E_{x}\left[u\left(X_{T}\right) ; T<\zeta\right] \\
& \geq c P_{x}[T=\zeta]+E_{x}\left[u^{c}\left(X_{T}\right) ; T<\zeta\right]-E_{x}\left[u\left(X_{T}\right) ; T<\zeta\right] \\
& \geq c P_{x}[T=\zeta]
\end{aligned}
$$

for any $T \in \mathcal{T}(x)$. Thus $\underline{D} u(x) \geq c$, and this yields $f^{*}(x) \leq \underline{D} u(x)$. In order to prove the converse inequality, we take $c>0$ such that $f^{*}(x)<c$ and define $T_{c} \in \mathcal{T}(x)$ as the first exit time from the open neighborhood $\left\{f^{*}<c\right\}$ of $x$. Then

$u(x)<R u^{c}(x)=E_{x}\left[\sup _{0 \leq t<\zeta} f^{*}\left(X_{t}\right) \vee c\right]=c P_{x}\left[T_{c}=\zeta\right]+E_{x}\left[\sup _{T_{c} \leq t<\zeta} f^{*}\left(X_{t}\right) ; T_{c}<\zeta\right]=c P_{x}\left[T_{c}=\zeta\right]+P_{T_{c}} u(x)$.

Since $u$ is excessive, this yields

$$
0 \leq u(x)-P_{T_{c}} u(x)<c P_{x}\left[T_{c}=\zeta\right]
$$

and in particular $P_{x}\left[T_{c}=\zeta\right]>0$, hence $\underline{D} u(x)<c$. This shows $\underline{D} u(x) \leq f^{*}(x)$.

\section{The Minimal And THE MAXIMAL REPRESENTING FUNCTION}

In this section we discuss the question to which extent a representing function $f$ is determined by the given excessive function $u$. For this purpose we introduce the notation

$$
\left.\widetilde{P}_{T} g(x):=E_{x}\left[g\left(X_{T}\right) ; T<\zeta\right]+E_{x} \varlimsup_{t \uparrow \zeta} g\left(X_{t}\right) ; T=\zeta\right]
$$

Note that

$$
\widetilde{P}_{T} u^{c}(x):=E_{x}\left[u^{c}\left(X_{T}\right) ; T<\zeta\right]+c P_{x}[T=\zeta]
$$

for any $c \geq 0$ due to condition (4).

Theorem 4.1. Suppose that $u$ admits the representation

$$
u(x)=E_{x}\left[\sup _{0<t<\zeta} f\left(X_{t}\right)\right]
$$

for any $x \in S$, where $f$ is regular on $S$. Then $f$ satisfies the bounds

$$
f_{*} \leq f \leq f^{*}=\underline{D} u,
$$

where the function $f_{*}$ is defined by

$$
f_{*}(x):=\inf \left\{c \geq 0 \mid \exists T \in \mathcal{T}(x): \widetilde{P}_{T} u^{c}(x) \geq u(x)\right\}
$$

for any $x \in S$.

Proof. For any $T \in \mathcal{T}(x)$ we get

$u(x)-P_{T} u(x)=E_{x}\left[\sup _{0<t<\zeta} f\left(X_{t}\right)\right]-E_{x}\left[\sup _{T<t<\zeta} f\left(X_{t}\right) ; T<\zeta\right] \geq E_{x}\left[\sup _{0<t<\zeta} f\left(X_{t}\right) ; T=\zeta\right] \geq f(x) P_{x}[T=\zeta]$ 
due to our assumption (14) on $f$, hence $f(x) \leq \underline{D} u(x)$. In order to verify the lower bound, take $c>f(x)$ and let $T_{c} \in \mathcal{T}(x)$ denote the first exit time from $\{f<c\}$. Since

$$
c \leq \sup _{T_{c}<t<\zeta} f\left(X_{t}\right)=\sup _{0<t<\zeta} f\left(X_{t}\right) \quad P_{x}-a . s . \text { on }\left\{T_{c}<\zeta\right\}
$$

due to property $(15)$ of $f$, we obtain

$$
\begin{aligned}
\widetilde{P}_{T_{c}} u^{c}(x)= & E_{x}\left[u^{c}\left(X_{T_{c}}\right) 1_{\left\{T_{c}<\zeta\right\}}+c 1_{\left\{T_{c}=\zeta\right\}}\right]=E_{x}\left[\sup _{T_{c}<t<\zeta} f\left(X_{t}\right) 1_{\left\{T_{c}<\zeta\right\}}+c 1_{\left\{T_{c}=\zeta\right\}}\right] \\
& \geq E_{x}\left[\sup _{0<t<\zeta} f\left(X_{t}\right)\right]=u(x),
\end{aligned}
$$

hence $c \geq f_{*}(x)$. This yields $f_{*}(x) \leq f(x)$.

The following example shows that the two bounds for the representing function $f$ in Theorem 4.1 may both be strict. In particular, the representing function may not be unique.

Example 4.1. Consider a Brownian motion on the interval $S=(0,3)$ and an upper-semicontinuous function $f$ on $S$ with $f(1)=f(2)=1$ which equals zero in $(0,1) \cup(2,3)$ and takes values in $(0,1)$ for $x \in(1,2)$. Denoting by $T_{b}:=\inf \left\{t \geq 0 \mid X_{t}=b\right\}$ the first passage time at level $b$, we can write

$$
\sup _{0<t<\zeta} f\left(X_{t}\right)=1_{(0,1)}(x) 1_{\left\{T_{1}<T_{0}\right\}}+1_{[1,2]}(x)+1_{(2,3)}(x) 1_{\left\{T_{2}<T_{3}\right\}} P_{x}-\text { a.s. }
$$

This shows that the excessive function $u$ defined by (1) is given by

$$
u(x)=\left\{\begin{array}{cl}
x & , x \in(0,1) \\
1 & , x \in[1,2] \\
3-x & , x \in(2,3)
\end{array} .\right.
$$

In this one-dimensional situation $R u^{c}$ coincides with the concave envelope of $u^{c}=u \vee c$ on $S$. Thus we obtain $f^{*}(x)=1_{[1,2]}(x)$ due to (11). Moreover, $f_{*}(x)=1_{\{1,2\}}(x)$ by inspection, hence $f_{*}(x)<f(x)<f^{*}(x)$ for $x \in(1,2)$. Note also that $f$ is regular since $f(x) \leq \sup _{0<t<\zeta} f\left(X_{t}\right) P_{x}-$ a.s. for any $x \in S$.

We are now going to derive an alternative description of $f_{*}$ which will allow us to identify $f_{*}$ as the minimal representing function for $u$.

Definition 4.1. Let us say that a point $x_{0} \in S$ is harmonic for $u$ if the mean-value property

$$
u\left(x_{0}\right)=E_{x_{0}}\left[u\left(X_{T_{\epsilon}}\right)\right]
$$

holds for $x_{0}$ and for some $\epsilon>0$, where $T_{\epsilon}$ denotes the first exit time from the ball $U_{\epsilon}\left(x_{0}\right)$. We denote by $H$ the set of all points in $S$ which are harmonic with respect to $u$.

From now on we assume that balls are regular in the following sense:

The exit laws from balls, defined as $\mu_{x}^{U}:=P_{x} \circ T_{\epsilon}^{-1}$ for $x \in U:=U_{\epsilon}\left(x_{0}\right)$, have the following properties:

A3) $\mu_{x}^{U} \approx \mu_{y}^{U}$ for all $x, y \in U$.

A4) If $U_{n}:=U_{\epsilon_{n}}\left(x_{0}\right)$ and $\epsilon_{n} \downarrow d\left(x_{0}, x_{1}\right)$ then $\mu_{x_{1}}^{U_{n}}$ converges weakly to $\delta_{x_{1}}$ as $n \uparrow \infty$.

Note that both assumptions are satisfied for $d$-dimensional Brownian motion. 
Lemma 4.1. $H$ coincides with the set of all points $x_{0} \in S$ such that $u$ is harmonic in some open neighborhood $G$ of $x_{0}$, i.e., the mean-value property

$u(x)=E_{x}\left[u\left(X_{T_{\epsilon}}\right)\right]$
holds for all $x \in G$ and all $\epsilon>0$ such that $\overline{U_{\epsilon}(x)} \subset G$. In particular $H$ is an open set.

Proof. If $u$ is harmonic in some open neighborhood $G$ of $x_{0}$ then the mean-value property (16) holds for $x_{0}$ and for $\epsilon$ small enough, and this shows $x_{0} \in H$. In order to prove the converse inclusion, we fix $x_{0} \in H$ and a corresponding $\epsilon>0$ such that $u\left(x_{0}\right)=E_{x_{0}}\left[u\left(X_{T_{\epsilon}}\right)\right]$. Then the function $h$ defined by $h(x):=E_{x}\left[u\left(X_{T_{\epsilon}}\right)\right]$ is harmonic on $U_{\epsilon}\left(x_{0}\right)$ and satisfies $h \leq u$ on $U_{\epsilon}\left(x_{0}\right)$ since $u$ is excessive. It remains to show that $u \geq h$ on $U_{\epsilon}\left(x_{0}\right)$. To this end, take $x_{1} \in U_{\epsilon}\left(x_{0}\right)$ and choose a sequence $0<\epsilon_{n}<\epsilon, n \in \mathbb{N}$, decreasing to $d\left(x_{0}, x_{1}\right)$. Denoting by $T_{\epsilon_{n}}$ the exit time from $U_{n}:=U_{\epsilon_{n}}\left(x_{0}\right)$, we obtain

$$
u\left(x_{0}\right) \geq E_{x_{0}}\left[u\left(X_{T_{\epsilon_{n}}}\right)\right] \geq E_{x_{0}}\left[h\left(X_{T_{\epsilon_{n}}}\right)\right]=E_{x_{0}}\left[E_{X_{T_{\epsilon_{n}}}}\left[u\left(X_{T_{\epsilon}}\right)\right]\right]=E_{x_{0}}\left[u\left(X_{T_{\epsilon}}\right)\right]=u\left(x_{0}\right) .
$$

This implies $u\left(X_{T_{\epsilon_{n}}}\right)=h\left(X_{T_{\epsilon_{n}}}\right) P_{x_{0}}-$ a.s., hence $P_{x_{1}}-$ a.s. due to our assumption A3). Thus

$$
h\left(x_{1}\right)=E_{x_{1}}\left[h\left(X_{T_{\epsilon_{n}}}\right)\right]=E_{x_{1}}\left[u\left(X_{T_{\epsilon_{n}}}\right)\right] .
$$

Using (4) and A4) we can conclude

$$
h\left(x_{1}\right)=\lim _{n \uparrow \infty} E_{x_{1}}\left[u\left(X_{T_{\epsilon_{n}}}\right)\right]=\lim _{n \uparrow \infty} \int u \mathrm{~d} \mu_{x_{1}}^{U_{n}}=u\left(x_{1}\right),
$$

since $u$ is continuous and bounded on $\bar{U}_{1}$.

Proposition 4.1. For any $x \in S$,

$$
f_{*}(x)=f^{*}(x) 1_{H^{c}}(x) .
$$

In particular $f_{*}$ is upper-semicontinuous. Moreover,

$$
f_{*}(x)=f^{*}(x)=\underline{D} u(x)=0
$$

for any $x \in H \backslash H_{0}$, where

$$
H_{0}:=\left\{x \in H \mid P_{x}\left[T_{H^{c}}<\zeta\right]=1\right\} .
$$

Proof. 1) For $x \in H$ there exists $\epsilon>0$ such that $\overline{U_{\epsilon}(x)} \subset S$ and $u(x)=E_{x}\left[u\left(X_{T_{\epsilon}}\right)\right]=\widetilde{P}_{T_{\epsilon}} u^{0}(x)$, and this implies $f_{*}(x)=0$. Now suppose that $x \in H^{c}$, i.e., $u$ is not harmonic in $x$. Note first that

$$
u(x)>E_{x}\left[u\left(X_{T}\right) ; T<\zeta\right]
$$

for any $T \in \mathcal{T}(x)$. Indeed, if $T$ is the first exit time from some open neighborhood $G$ of $x$ then

$$
E_{x}\left[u\left(X_{T}\right) ; T<\zeta\right]=E_{x}\left[E_{X_{T_{\epsilon}}}\left[u\left(X_{T}\right) ; T<\zeta\right]\right] \leq E_{x}\left[u\left(X_{T_{\epsilon}}\right)\right]<u(x)
$$

for any $\epsilon>0$ such that $\overline{U_{\epsilon}(x)} \subseteq G$. In view of Theorem 4.1 we have to show $f_{*}(x) \geq f^{*}(x)$, and we may assume $f^{*}(x)>0$. Choose $c>0$ such that $f^{*}(x)>c$. Then there exists $\epsilon>0$ such that $R u^{c+\epsilon}(x)=u(x)$, i.e.,

$$
\widetilde{P}_{T} u^{c+\epsilon}(x) \leq u(x)
$$

for any $T \in \mathcal{T}(x)$ in view of (8). Fix $\delta \in(0, \epsilon)$ and $T \in \mathcal{T}(x)$. If $T<\zeta$ and $u\left(X_{T}\right) \geq c+\delta P_{x}-$ a.s. then

$$
\widetilde{P}_{T} u^{c+\delta}(x)=E_{x}\left[u\left(X_{T}\right) ; T<\zeta\right]<u(x)
$$


due to (19). On the other hand, if $P_{x}[T=\zeta]+P_{x}\left[u\left(X_{T}\right)<c+\delta ; T<\zeta\right]>0$ then

$$
\widetilde{P}_{T} u^{c+\delta}(x)<\widetilde{P}_{T} u^{c+\epsilon}(x) \leq u(x)
$$

due to (20). Thus we obtain $u(x)>\widetilde{P}_{T} u^{c+\delta}(x)$ for any $T \in \mathcal{T}(x)$, hence $f_{*}(x) \geq c+\delta$. This concludes the proof of (17). Upper-semicontinuity of $f_{*}$ follows immediately since $f^{*}$ is upper-semicontinuous and $H^{c}$ is closed.

2) Take $x \in H \backslash H_{0}$ and consider a sequence $G_{n}, n \in \mathbb{N}$, of relatively compact open neighborhoods of $x$ such that $G_{n} \nearrow H$ as $n \uparrow \infty$. Let $T_{n}:=T_{G_{n}^{c}}$ denote the first exit time from $G_{n}$. Then $T_{n} \nearrow T_{H^{c}}$, hence $X_{T_{n}}$ converges to $X_{T_{H^{c}}} P_{x}-$ a.s. on $\left\{T_{H^{c}}<\zeta\right\}$ due to the quasi-left-continuity of our Hunt process $\left(X_{t}\right)_{t \geq 0}$. But this shows

$$
u(x)=\lim _{n \uparrow \infty} E_{x}\left[u\left(X_{T_{n}}\right)\right]=E_{x}\left[\lim _{n \uparrow \infty} u\left(X_{T_{n}}\right)\right]=E_{x}\left[u\left(X_{T_{H^{c}}}\right) ; T_{H^{c}}<\zeta\right]=P_{T_{H^{c}}} u(x)
$$

in view of our assumptions (3) and (4) on $u$. Since $T_{H^{c}} \in \mathcal{T}(x)$ satisfies $P_{x}\left[T_{H^{c}}=\zeta\right]>0$ for $x \in H \backslash H_{0}$, we obtain

$$
0 \leq f_{*}(x) \leq f^{*}(x)=\underline{D} u(x) \leq \frac{u(x)-P_{T_{H^{c}}} u(x)}{P_{x}\left[T_{H^{c}}=\zeta\right]}=0 .
$$

Our next goal is to show that $u$ admits a representation in terms of $f_{*}$.

Lemma 4.2. For any stopping time $T_{0}$ the following conditions are satisfied $P_{x}-$ a.s. on $\left\{T_{0}<\zeta\right\} \cap\left\{X_{T_{0}} \in H_{0}\right\}$ :

i) $T_{1}:=T_{0}+T_{H^{c}} \circ \theta_{T_{0}}<\zeta$.

ii) $f^{*}\left(X_{T_{1}}\right)=\sup _{T_{0}<t \leq T_{1}} f^{*}\left(X_{t}\right)$.

Proof. Since the exit time $T:=T_{H^{c}}$ from $H$ satisfies $P_{y}\left[T_{H^{c}}<\zeta\right]=1$ for any $y \in H_{0}$, the first assertion follows from

$$
P_{x}\left[\left\{T_{1}<\zeta\right\} \cap\left\{T_{0}<\zeta, X_{T_{0}} \in H_{0}\right\}\right]=E_{x}\left[P_{X_{T_{0}}}[T<\zeta] ; T_{0}<\zeta, X_{T_{0}} \in H_{0}\right] .
$$

In order to verify property ii), note that

$$
P_{x}\left[f^{*}\left(X_{T_{1}}\right)=\sup _{T_{0}<t \leq T_{1}} f^{*}\left(X_{t}\right) ; T_{0}<\zeta, X_{T_{0}} \in H_{0}\right]=E_{x}\left[P_{X_{T_{0}}}\left[f^{*}\left(X_{T}\right)=\sup _{0<t \leq T} f^{*}\left(X_{t}\right)\right] ; T_{0}<\zeta, X_{T_{0}} \in H_{0}\right] .
$$

It is therefore enough to show that

$$
\Lambda_{T}^{*}:=\sup _{0<t \leq T} f^{*}\left(X_{t}\right)=f^{*}\left(X_{T}\right) \quad P_{y}-a . s .
$$

for any $y \in H_{0}$. Clearly, we have $f^{*}\left(X_{T}\right) \leq \Lambda_{T}^{*}$. Since $T<\zeta P_{y}-$ a.s., the representation (12) allows us to conclude

$$
\begin{aligned}
u(y) & =E_{y}\left[\sup _{0<t<\zeta} f^{*}\left(X_{t}\right)\right]=E_{y}\left[E_{X_{T}}\left[\Lambda_{T}^{*} \vee \sup _{0<t<\zeta} f^{*}\left(X_{t}\right)\right]\right] \\
& =E_{y}\left[R u^{\Lambda_{T}^{*}}\left(X_{T}\right)\right] \geq E_{y}\left[u\left(X_{T}\right)\right]=u(y),
\end{aligned}
$$

i.e., $E_{y}\left[R u^{\Lambda_{T}^{*}}\left(X_{T}\right)\right]=E_{y}\left[u\left(X_{T}\right)\right]$. In view of $R u^{\Lambda_{T}^{*}}\left(X_{T}\right) \geq u\left(X_{T}\right)$ this implies $R u^{\Lambda_{T}^{*}}\left(X_{T}\right)=u\left(X_{T}\right) P_{y}-a . s$. or, equivalently, $f^{*}\left(X_{T}\right) \geq \Lambda_{T}^{*} P_{y}-$ a.s.

We are now ready to prove that $f_{*}$ is the minimal representing function for $u$. 
Proposition 4.2. For any $x \in S$ and any upper-semicontinuous function $f$ such that $f_{*} \leq f \leq f^{*}$,

$$
\sup _{0<t<\zeta} f_{*}\left(X_{s}\right)=\sup _{0<t<\zeta} f\left(X_{t}\right)=\sup _{0<t<\zeta} f^{*}\left(X_{t}\right) \quad P_{x}-\text { a.s. },
$$

and so $f$ is a regular representing function for $u$. In particular we obtain the representation

$$
u(x)=E_{x}\left[\sup _{0<t<\zeta} f_{*}\left(X_{t}\right)\right],
$$

and $f_{*}$ is the minimal regular function yielding a representation of $u$.

Proof. Let us first prove (21) for $x \in H$. Since $0 \leq f_{*} \leq f \leq f^{*}$, it is enough to show that $\sup _{0<t<\zeta} f_{*}\left(X_{t}\right) \geq c$ $P_{x}-$ a.s. on $\left\{T_{c}<\zeta\right\}$ for fixed $c>0$, where $T_{c}$ denotes the exit time from the open set $\left\{f^{*}<c\right\}$. Note first that

$$
\sup _{0<t<\zeta} f_{*}\left(X_{t}\right) \geq f_{*}\left(X_{T_{c}}\right)=f^{*}\left(X_{T_{c}}\right) \geq c \quad P_{x}-\text { a.s. on }\left\{T_{c}<\zeta\right\} \cap\left\{X_{T_{c}} \in H^{c}\right\},
$$

due to (17). On $\left\{T_{c}<\zeta\right\} \cap\left\{X_{T_{c}} \in H\right\}$ we have $X_{T_{c}} \in H_{0} P_{x}-a . s$. due to (18) since $f^{*}\left(X_{T_{c}}\right) \geq c>0$. Lemma 4.2 allows us to conclude that $T_{1}:=T_{c}+T_{H^{c}} \circ \theta_{T_{c}}$ satisfies $T_{1}<\zeta P_{x}-$ a.s. and

$$
\begin{aligned}
\sup _{0<t<\zeta} f_{*}\left(X_{t}\right) & \geq f_{*}\left(X_{T_{1}}\right)=f^{*}\left(X_{T_{1}}\right) \\
& =\sup _{T_{c}<t \leq T_{1}} f^{*}\left(X_{t}\right) \geq f^{*}\left(X_{T_{c}}\right) \geq c \quad P_{x}-\text { a.s. on }\left\{T_{c}<\zeta\right\} \cap\left\{X_{T_{c}} \in H\right\}
\end{aligned}
$$

due to (17). This concludes the proof of (21) for $x \in H$. In particular, we obtain

$$
\sup _{\tilde{T}<t<\zeta} f_{*}\left(X_{t}\right)=\sup _{\widetilde{T}<t<\zeta} f\left(X_{t}\right)=\sup _{\widetilde{T}<t<\zeta} f^{*}\left(X_{t}\right) \quad P_{x}-a . s . \text { on }\left\{\widetilde{T}<\zeta, X_{\widetilde{T}} \in H\right\}
$$

for any stopping time $\widetilde{T}$, due to the strong Markov property.

It remains to prove (21) for $x \in H^{c}$. To this end, we denote by $\widehat{T}$ the first exit time from $H^{c}$. Since, by Proposition $4.1, f_{*}$ and $f^{*}$ coincide on $H^{c}$, the identity (21) holds on the set $\{\widehat{T}=\zeta\}$. On the other hand, using again Proposition 4.1, we have

$$
\begin{aligned}
\sup _{0<t<\zeta} f^{*}\left(X_{t}\right) \vee u_{\zeta} & =\sup _{0<t \leq \widehat{T}} f^{*}\left(X_{t}\right) \vee \sup _{\widehat{T}<t<\zeta} f^{*}\left(X_{t}\right) \vee u_{\zeta} \\
& =\sup _{0<t \leq \widehat{T}} f_{*}\left(X_{t}\right) \vee \sup _{\widehat{T}<t<\zeta} f^{*}\left(X_{t}\right) \vee u_{\zeta} \quad \text { on }\{\widehat{T}<\zeta\} .
\end{aligned}
$$

By definition of $\widehat{T}$, on $\{\widehat{T}<\zeta\}$ there exists a sequence of stopping times $\widehat{T}<T_{n}<\zeta, n \in \mathbb{N}$, decreasing to $\widehat{T}$ such that $X_{T_{n}} \in H$, and so we can conclude that

$$
\begin{aligned}
\sup _{\widehat{T}<t<\zeta} f^{*}\left(X_{t}\right) \vee u_{\zeta} & =\lim _{n \uparrow \infty} \sup _{T_{n}<t<\zeta} f^{*}\left(X_{t}\right) \vee u_{\zeta} \\
& =\lim _{n \uparrow \infty} \sup _{T_{n}<t<\zeta} f_{*}\left(X_{t}\right) \vee u_{\zeta} \\
& =\sup _{\widehat{T}<t<\zeta} f_{*}\left(X_{t}\right) \vee u_{\zeta} \quad P_{x}-\text { a. s. on }\{\widehat{T}<\zeta\}
\end{aligned}
$$

due to (22). Combined with (23) this yields $(21)$ on $\{\widehat{T}<\zeta\}$. Thus we have shown that (21) holds as well for any $x \in H^{c}$. 
In particular $f$ is a representing function for $u$. Moreover,

$$
f(x) \leq f^{*}(x) \leq \sup _{0<t<\zeta} f^{*}\left(X_{t}\right)=\sup _{0<t<\zeta} f\left(X_{t}\right) \quad P_{x}-a . s .
$$

for any $x \in S$ due to (21), and so $f$ is a regular function on $S$.

Corollary 4.1. Let $f$ be any regular representing function for $u$. Then

$$
R u^{c}(x)=E_{x}\left[\sup _{0<t<\zeta} f\left(X_{t}\right) \vee c\right]
$$

for any $x \in S$ and for any $c \geq 0$.

Proof. In view of (21) the claim follows immediately from the representation (12) of $R u^{c}$.

Remark 4.1. Let us go back to the simple Example 4.1 in order to illustrate the preceding results. Here the set $H$ of harmonic points for $u$ is given by $(0,1) \cup(1,2) \cup(2,3)$. Our observation above that $f_{*}=0$ on $H$ and $f_{*}=f^{*}$ on $H^{c}=\{1,2\}$ is explained by the general Proposition 4.1. Moreover, we have $H_{0}=(1,2)$ and $f_{*}=f^{*}=0$ on $H \backslash H_{0}=(0,1) \cup(2,3)$, in accordance with equation (18).

\section{REFERENCES}

[1] P. Bank and N. El Karoui, A Stochastic Representation Theorem with Applications to Optimization and Obstacle Problems. Ann. Probab. 32 (2005) 1030-1067.

[2] P. Bank and H. Föllmer, American Options, Multi-armed Bandits, and Optimal Consumption Plans: A Unifying View, in Paris-Princeton Lectures on Mathematical Finance 2002, Lect. Notes Math. 1814 (2003) 1-42.

[3] C. Dellacherie and P. Meyer, Probabilités et potentiel. Chapitres XII-XVI: Théorie du potentiel associée à une résolvante, Théorie des processus de Markov, Hermann, Paris (1987).

[4] N. El Karoui, Les aspects probabilistes du contrôle stochastique, in Ninth Saint Flour Probability Summer School-1979 (Saint Flour, 1979), Lect. Notes Math. 876 (1981) 73-238.

[5] N. El Karoui, Max-Plus Decomposition of Supermartingale - Application to Portfolio Insurance, http://www. ima.umn.edu/talks/workshops/4-12-16.2004/el_karoui/IMA2004.pdf (2004).

[6] N. El Karoui and H. Föllmer, A non-linear Riesz representation in probabilistic potential theory, in Annales de l'Institut Henri Poincaré (B) Probabilités et Statistiques 41 (2005) 269-283.

[7] N. El Karoui and A. Meziou, Constrained optimization with respect to stochastic dominance: Application to portfolio insurance. Math. Finance 16 (2006) 103-117.

[8] T. Knispel, Eine nichtlineare Riesz-Darstellung bezüglich additiver Funktionale im potentialtheoretischen Kontext. Diploma Thesis, Humboldt University, Berlin (2004).

[9] A.N. Shiryaev, Statistical Sequential Analysis. AMS, Providence, Transl. Math. Monographs 38 (1973). 